\title{
A simple approach to determine the best threshold value for automatic image thresholding
}

\begin{abstract}
Image thresholding is a powerful yet simple method to highlight the subject from its background in image scene analysis. Lots of methods have been proposed around the globe while some researchers regard this matter as a non-trivial problem. This paper proposes a simple approach for fast calculation of the threshold value for automatic image thresholding based on gradient analysis of the image histogram. The method manages to successfully differentiate the subject from the background. The proposed approach is validated by illustrative examples. Satisfactory results were acquired with other methods that use more complex algorithms.
\end{abstract}

